\title{
КВАНТОВОЕ ИЗМЕНЕНИЕ ПОЛЯРИЗАЦИИ СВЕТА КОЛЛЕКТИВОМ ОРИЕНТИРОВАННЫХ АТОМОВ
}

\author{
V. HIZNIAKOV, M. ROZMAN. VALGUSE POLARISATSIOONI KVANTMUUTUS ORIENTEERITUD \\ AATOMITE KOLLEKTIIVI TOIMEL \\ V. HIZHNYAKOV, M. ROZMAN. COOPERATIVE QUANTUM EFFECT OF ORIENTED ATOMS ON \\ LIGHT POLARIZATION
}

1. Данная работа посвящена изучению взаимодействия коллектива ориентированных атомов с когерентным поляризованным световым импульсом. Исследуется поляризация прошедших (не рассеянных) фотонов. Подобная задача в случае одного атома рассматривалась ранее в [ $\left.{ }^{1}\right]$. Было показано, что в процессе взаимодействия частота фотонов перенормируется. Это приводит к конечному приращению фазы взаимодействующей с атомом поляризационной компоненты излучения, вследствие чего линейная поляризация фотонов изменяется на эллиптическую. При соответствующей дифракционному пределу полной фокусировке излучения на атом приращение фазы может достигать величин $\sim 10^{\circ}\left[{ }^{1}\right]$. Ниже будет показано, что в случае коллектива тождественных атомов это приращение фазы может быть и большим: для достаточно длинных световых импульсов оно линейно возрастает с увеличением числа атомов.

2. Рассмотрим две моды излучения одинаковой частоты $\omega$, но разной поляризации и $N_{0}$ одинаковых двухуровневых атомов, помещенных в объем с линейными размерами, меньшими, чем длина волны света $\lambda=2 \pi^{0} c \omega^{-1}$. Поляризации мод таковы, что одна из них не взаимодействует с атомом, а другая взаимодействует. Гамильтониан этой системы имеет вид

где

$$
H=\omega\left(\hat{N}+\hat{N}_{\perp}\right)+H_{1}
$$

$$
H_{1}=2 \Delta S_{z}+\gamma\left(S_{+} a_{\|}+S_{-} a_{\|}^{+}\right) \text {, }
$$

$\hat{N}=\hat{N}_{\|}+S_{+} S_{-}, \hat{N}_{\perp(\|)}=a_{\perp(\|)}^{+} a_{\perp(\|)}-$ оператор числа фотонов, $a_{\perp(\text { (I) }}$ и $a_{\perp(\|)}-$ операторы рождения и уничтожения фотонов невзаимодействующей (взаимодействующей) моды, $S_{ \pm}=S_{x} \pm i S_{y}, S_{\alpha}$ - декартовы компоненты вектора квазиспина $(\alpha=x, y, z)\left[{ }^{2}\right] ; \Delta=\left(\omega_{0}-\omega\right) / 2, \omega_{0}-$ частота атомных возбуждений, $\gamma-$ константа взаимодействия атома с модой $\|, \hbar=1$. Гамильтонианы $H$ и $H_{1}$ коммутируют с операторами $\hat{N}$ и $\hat{N}_{\perp}$. Поэтому число фотонов плюс число атомных возбуждений (число частиц) сохраняется. Классифицируемые по этому квантовому числу собственные состояния гамильтониана $H_{1}$ называются одетыми состояниями.

Рассмотрим подпространство одетых состояний, характеризующееся 
большим числом частиц $N \gg N_{0}$. Используем в качестве базиса состояния $|m\rangle=|S, m\rangle|N-S-m\rangle_{\|}$, где $|N\rangle_{\|}-N$-фотонные состояния моды $\|, \mid S, m>-$ коллективные состояния Дикке $\left[{ }^{2}\right]$, описывающие систему $N_{0}$ двухуровневых атомов с суммарным квазиспином $S$ и его проекцией на ось $z$ равную $m ; S=N_{0} / 2, N_{0} / 2-1, \ldots, 1 / 2(0)$, $m=-S,-S+1, \ldots, S$. (Если все атомы возбуждены, то $S=m=N_{0} / 2$, а если не возбуждены, то $\left.S=-m=N_{0} / 2\right)$. В этом базисе гамильтониан $H_{1}$ имеет вид трехдиагональной матрицы с отличными от нуля элементами $\left(H_{1}\right)_{m, m}=2 \Delta m,\left(H_{1}\right)_{m, m+1}=\left(H_{1}\right)_{m+1, m}=\gamma C_{m}\left(N_{1}-m\right)^{1 / 2}$, где $C_{m}=$ $=[(S-m)(S+m+1)]^{1 / 2}, N_{1}=N-S$. В данном случае $N \gg N_{0} \geqslant 2 S$ можно принять $\left(H_{1}\right)_{m, m+1} \approx \Gamma C_{m}(1-m / 2 N)$, где $\Gamma=\gamma N^{1 / 2}$. В этом приближении

$$
H_{1}=2 \Delta S_{z}+2 \Gamma S_{x}-\frac{\Gamma}{N} S_{z} S_{x}
$$

причем последнее слагаемое можно рассматривать как малое возмущение.

Перейдем к новому представлению с помощью унитарного преобразования поворота вокруг оси $y$ на угол $\varphi=\arctan (\Gamma / \Delta)$. В этом представлении

$$
H_{1}=2 \Omega \sigma_{z}-\frac{\Gamma}{2 N}\left[\sigma_{x} \sigma_{z} \cos 2 \varphi+i \sigma_{y} \cos ^{2} \varphi+\left(\sigma_{x}^{2}-\sigma_{z}^{2}\right) \sin 2 \varphi\right],
$$

где $\Omega=\left(\Delta^{2}+\Gamma^{2}\right)^{1 / 2}$ - частота Раби, $\sigma_{\alpha}$ - компіоненты векторного оператора квазиспина в повернутой системе отсчета; правила коммутации и собственные значения операторов $\sigma_{\alpha}$, естественно, те же что и для $S_{\alpha}$. Поэтому собственные значения $H_{1}$ в первом приближении по $\Gamma / N$ равны

$$
E_{S, M}=2 \Omega M+\frac{\Gamma \sin 2 \varphi}{4 N}\left(S(S+1)-3 M^{2}\right), \quad M=-S, \ldots S .
$$

В этой формуле основным является первое слагаемое, второе дает лишь малую поправку. Следовательно, $N$-частичные одетые состояния образуют систему $2 S+1$ приближенно эквидистантных уровней. Расстояние между соседними уровнями практически такое же, как в случае одного атома, однако расстояние между крайними уровнями возрастает в $2 S$ раз.

3. Предположим, что до взаимодействия с атомами линейно поляризованный световой импульс находится в глауберовском когерентном состоянии

$$
|\alpha\rangle=e^{-|\alpha|^{2} / 2} \sum_{n, n^{\prime}} \frac{\alpha_{\|}^{n} \alpha_{\perp}^{n^{\prime}}}{\sqrt{n ! n^{\prime} !}}|n\rangle_{\|}\left|n^{\prime}\right\rangle_{\perp} e^{i \omega\left(n+n^{\prime}\right) t}, \quad t \rightarrow-\infty
$$

где $|n\rangle_{\|}$и $|n\rangle_{\perp} \quad-n$-фотонные состояния мод $\|$ и $\perp, t-$ время, $\alpha_{\|}=\alpha \cos \beta, \alpha_{\perp}=\alpha \sin \beta, \beta-$ угол между векторами поляризации возбужденной моды и моды $\|,|\alpha|^{2}=\bar{N}-$ среднее число фотонов; $\bar{N} \gg N_{0}$. Предположим также, что до взаимодействия атомы не возбуждены, г. е. заняты уровни с $M=S=N_{0} / 2$, если $\Delta>0$, либо уровни с $M=$ $=-S=-N_{0} / 2$, если $\Delta<0$. Считаем, что спектральная ширина импульса $\Delta \omega$ мала $(\Delta \omega \ll \Delta \leqslant \Omega)$. В таком случае реализуется адиабатически медленный режим включения и выключения взаимодействия (т. е. $\gamma=\gamma(t)$ есть медленная функция времени). Поэтому после прохождения импульсом атомов последние останутся в основном состоянии, а импульс перейдет в состояние 


$$
|\bar{\alpha}\rangle=e^{-|\alpha|^{2 / 2}} \sum_{n, n^{\prime}} \frac{\alpha_{\|}^{n} \alpha_{\perp}^{n^{\prime}}}{\sqrt{n ! n^{\prime} !}}|n\rangle_{\|}\left|n^{\prime}\right\rangle_{\perp} e^{-i \varphi_{n}-i \omega\left(n+n^{\prime}\right) t}, \quad t \rightarrow \infty
$$

где приращения фаз $\varphi_{n}$ определяются интегралом энергий уровней $E_{S, M}, S=N_{0} / 2= \pm M$ по времени взаимодействия $\left[{ }^{3}\right]$ :

$$
\varphi_{n}= \pm N_{0} \int_{-\infty}^{\infty} \Omega(t) d t= \pm N_{0} \int_{-\infty}^{\infty}\left[\Delta^{2}+\left(n-N_{0} / 2\right) \gamma^{2}(t)\right]^{1 / 2} d t
$$

$\left(n \geqslant N_{0} / 2\right)$. Определим разность фаз мод $\|$ и $\perp$ в состоянии

$$
\begin{gathered}
\Delta \varphi=i \ln \left\langle\bar{\alpha}\left|e^{-i \varphi_{\perp}} e^{i \varphi_{\|}}\right| \bar{\alpha}\right\rangle= \\
=-|\alpha|^{2}+i \ln \sum_{n, n^{\prime}} \frac{\left|\alpha_{\|}\right|^{2 n-1}\left|\alpha_{\perp}\right|^{2 n^{\prime}+1}}{(n-1) ! n^{\prime} ! \sqrt{n\left(n^{\prime}+1\right)}} e^{-i\left(\varphi_{n}-\varphi_{n-1}\right)}
\end{gathered}
$$

где $e^{i \hat{\varphi}}=\left(a^{+} a+\perp\right)^{-1 / 2} a-$ оператор фазы. Учитывая, что основной вклад в суммы по $n$ и $n^{1}$ дают слагаемые с большими $n$ и $n^{\prime}$ $\left(n \approx\left|\alpha_{\|}\right|^{2} \sim \bar{N} \gg N_{0}, \quad n^{\prime} \approx\left|\alpha_{\perp}\right|^{2} \sim \bar{N} \gg 1\right)$, находим

где

$$
\Delta \varphi \approx \pm N_{0} \varphi_{0}
$$

$$
\varphi_{0}=\int_{-\infty}^{\infty} \frac{\gamma^{2}(t) d t}{\sqrt{\Delta^{2}+\bar{N} \cos ^{2} \beta \cdot \gamma^{2}(t)}} .
$$

Таким образом, после прохождения когерентным световым импульсом атомов возникает разность фаз \| и $\perp$ компонент поляризации, что соответствует превращению линейно поляризованного света в эллиптически поляризованный. Отмеченная разность линейно растет с ростом числа атомов $N_{0}$.

4. В приведенном выше анализе не учитывалось радиационное затухание возбужденных состояний атомов. Это оправдано в том случае, когда частота Раби велика по сравнению с радиационной шириной возбужденного уровня атома $\gamma_{0}\left(\Omega \gg \gamma_{0}\right)$. Использованные выше монохроматическое и диабатическое приближения предполагают также выполнимость условия $\Delta \omega \ll \Omega$. В $\left[{ }^{1}\right]$ показано, что эти условия ограничивают $\varphi_{0}$ величиной $\sim 3 \lambda / 2 \pi S^{1 / 2} \leqslant 3 / 2 \pi \approx 27^{\circ}$, где $S-$ сечение фокусировки. Поэтому в случае $N_{0}$ атомов

$$
|\Delta \varphi| \leqslant 3 N_{0} \lambda / 2 \pi S^{1 / 2} \leqslant 3 N_{0} / 2 \pi .
$$

Отсюда следует, что для большого числа атомов разность фаз мод ॥ и $\perp$ может принимать практически любые значения. Поэтому, если $\mathrm{N}_{0}$ существенно флуктуирует, то свет заметно деполяризуется. Деполяризация света должна происходить также в случае импульса с коротким передним и (или) задним фронтом вследствие неадиабатических эффектов при включении и выключении взаимодействия.

\section{Л ИТЕРА Т Р А} 1. Хижняков В. В., Розман М. Г. Изв. АН ЭССР. Физ. Матем., 34, № 2, 226-228
(1985).

2. Dicke, R. H. Phys. Rev., 93, № 1, 99-112 (1954).

3. Бом Д. Квантовая теория. М., ГЙФМЛ, 1961, 573.

Ннститут физики

Академии наук Эстонской ССР
Поступила в редакцию $24 /$ IV 1985 